\title{
Possible involvement of proteinaceous and non-proteinaceous trehalase inhibitors in the regulation of hemolymph trehalose concentration in Bombyx mori
}

\author{
Nujira Tatun, ${ }^{1}$ Tippawan Singtripor, ${ }^{1}$ Shingo Osugi, ${ }^{2}$ Siriluck NaChiangmai, ${ }^{1}$ \\ Masafumi IWAMI ${ }^{2}$ and Sho SAKURAI ${ }^{2, *}$ \\ ${ }^{1}$ Department of Biology, Faculty of Science, Chiang Mai University; Chiang Mai, 50200 Thailand \\ ${ }^{2}$ Division of Life Sciences, Graduate School of Natural Science and Technology, Kanazawa University; Kanazawa 920-1192, \\ Japan
}

(Received 24 June 2008; Accepted 24 September 2008)

\begin{abstract}
The midgut of the silkworm Bombyx mori contains two types of trehalase: soluble and membrane-bound. The trehalose concentration in larval hemolymph remains substantially high through the feeding period of the last (fifth) larval instar, despite appreciable trehalase activity through this period. We found that $B$. mori larval hemolymph contains two types of trehalase inhibitor, a proteinaceous inhibitor (inhibitor-P) and an inhibitor that is extractable with methanol and ethanol (inhibitor-M). Both inhibit the two types of trehalase, but with different sensitivities. Inhibitor-P inhibited soluble trehalase (ST) more effectively than membrane-bound trehalase (MT), while inhibitor-M inhibited MT slightly more than ST. The inhibitory activity of inhibitor-M was high during the early to middle feeding period, followed by a sudden decrease during the late feeding period. Inhibitor-P activity increased throughout the feeding period, and markedly decreased 2 days after gut purge. Thus, the two inhibitors exhibited stage-specific changes in the hemolymph, indicating coordinated inhibitory activity. Therefore, trehalose concentration regulation in the hemolymph appears to be complex, consisting of at least two inhibitors and two types of trehalase.
\end{abstract}

Key words: Soluble trehalase; membrane-bound trehalase; trehalase inhibitor; midgut; Bombyx mori

\section{INTRODUCTION}

Holometabolous insects actively consume and store nutrition during the last larval instar in preparation for the energy-demanding pupal stage, during which adult differentiation of tissues and gamete production occurs without exogenous energy sources. The energy is stored as fat, storage protein, and glycogen. In the silkworm Bombyx mori and other lepidopterans, incorporated fatty acids are converted to and stored as diacylglyceride in lipophorin, amino acids in storage proteins, and sugars in trehalose, mostly in the hemolymph (Wyatt, 1967; Chino et al., 1981; Mullins, 1985; Miller et al., 1990). Thus, hemocoel serves as a storage site for energy molecules. At the end of the feeding period, these materials are transferred to body fat, the major storage compound during the prepupal and pupal periods, in which diacylglyceride is converted to triacylglyceride and trehalose is stored as glycogen.

Trehalose $(\alpha$-D-glucopyranosyl-[1-1]- $\alpha$-D-glucopyranoside), a disaccharide consisting of two glucose molecules, is the major hemolymph sugar in all insects (Becker et al., 1996). Because it is membrane-impermeable, it is hydrolyzed by trehalase into glucose upon utilization by the tissues; one exception is the African chironomid Polypedilum vanderplanki, in which trehalose transporter facilitates trehalose incorporation (Kikawada et al., 2007). Insects have either membrane-bound trehalase or both soluble and membrane-bound forms (Thompson, 2003). Both types are present in B. mori (Sumida and Yamashita, 1983; Su et al., 1993; Mitsumasu et al., 2005), the Eri silkworm Samia cynthia ricini (Hirayama et al.,

* To whom correspondence should be addressed at: E-mail: ssakurai@kenroku.kanazawa-u.ac.jp DOI: 10.1303/aez.2009.85 
2007), and the bamboo borer Omphisa fuscidentalis (Tatun et al., 2008b). In addition, the enzymatic activities of both trehalases change during growth and development (Yamashita et al., 1974; Tatun et al., 2008b), indicating that trehalase regulation is involved in the translocation of stored sugars from the hemolymph to the body fat and vice versa.

Trehalose concentration in the hemolymph is regulated homeostatically in the short term (minutes and hours; Oda et al., 2000; Blatt and Roces, 2001) and long term (days and months) as seen in insect growth (Friedman, 1978; Ogiso and Takahashi, 1984; Oda et al., 1997) and diapause (Goto et al., 1993; Singtripop et al., 2002). Long-term regulation has been studied from the perspective of hormonal control of trehalase activities (Steele and Paul, 1985), i.e., hyperglycemic hormone for homeostatic regulation (Gäde et al., 1997) and 20hydroxyecdysone (Yaginuma and Happ, 1989; Oda et al., 2000; Tatun et al., 2008a) for regulation during larval growth and larval-pupal transformation. However, hormonal regulation appears to be an insufficient explanation for long-term changes in hemolymph trehalose concentration.

Figure 1 shows changes in trehalose concentration in the hemolymph and enzymatic activities of soluble and membrane-bound trehalases through the fifth instar and early pupal period of B. mori. In the early to middle fifth instar, trehalose remains at a stable concentration of about $10 \mathrm{~mm}$, and the net amount of trehalose in individual larvae increases by three- to four-fold during the first 4 days (Oda et al., 1997). During this period, membrane-bound trehalase activity in the midgut increases until Day 3 , and soluble trehalase activity begins to increase on Day 4 (Yamashita et al., 1974). The in vitro enzymatic activity of a whole gut preparation of Day 3 larvae is about $3 \mu \mathrm{mol} / \mathrm{min} / \mathrm{midgut}$ (Azuma and Yamashita, 1985), and the estimated total amount of trehalose in one Day 3 larva is about $10 \mu \mathrm{mol}$ (Oda et al., 1997). If the trehalase activity of the whole midgut in vitro is similar to that in vivo, trehalose would be cleared out within a few minutes even under the release of trehalose from fat bodies. Nevertheless, trehalose concentration remains at a stable level and the net amount increases, indicating that trehalase activity may be partially or mostly inhibited by an inhibitor(s) in the hemolymph, because trehalose is used for energy-de-

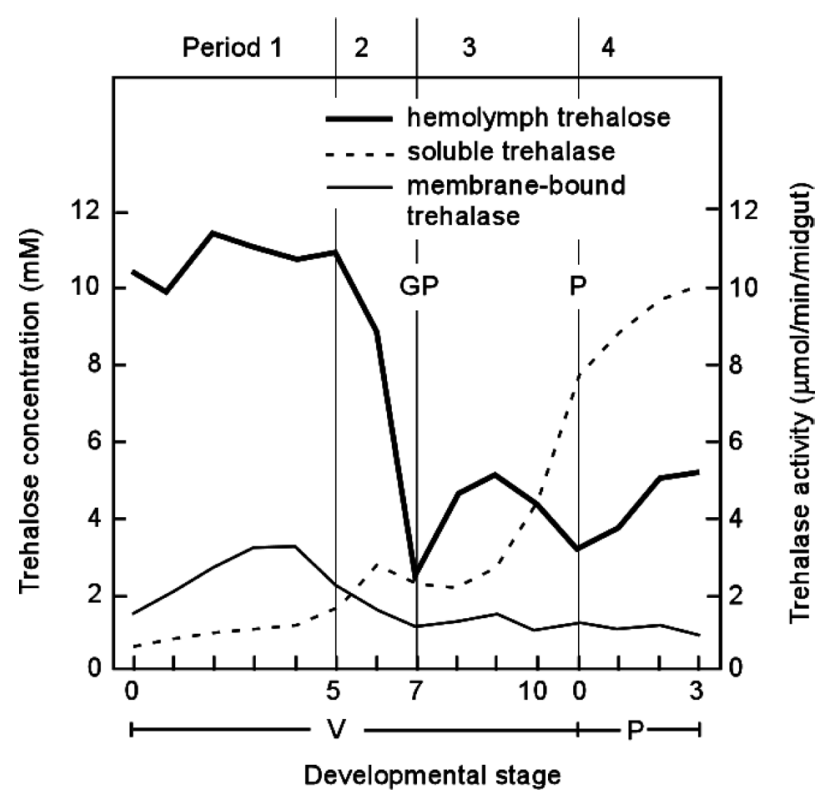

Fig. 1. Changes in trehalose concentration in the hemolymph (thick line), and enzymatic activities of membranebound trehalase (thin line) and soluble trehalase (broken line) in the fifth instar and early pupal period of $B$. mori. Trehalose concentrations and trehalase activities are modified from Oda et al. (1997), and Yamashita et al. (1974), respectively.

manding tasks such as muscle contraction and silk protein synthesis.

Trehalase inhibitors have been reported in microorganisms (Knuesel et al., 1998; Wegener et al., 2003), plants (Silva et al., 2006; Hirayama et al., 2007), and three insect species. Plants contain various trehalase inhibitors, which are non-proteinaceous molecules and function as defense against herbivorous insects (Hirayama et al., 2007). Leaves of mulberry, the host plant of $B$. mori, contain sugar-mimic alkaloids that act as trehalase inhibitors (Hirayama et al., 2007). The alkaloids are absorbed into the hemolymph, indicating that an exogenous trehalase inhibitor may be involved in trehalose regulation in the hemolymph, although there is no data to support this view. In contrast, insects produce trehalase inhibitors, all of which are proteinaceous; these have been reported from the blowfly Phormia regina (Friedman, 1961), the American cockroach Periplaneta americana (Hayakawa et al., 1989), and O. fuscidentalis (Tatun et al., 2008b). In a preliminary study, we found trehalase inhibitor in the hemolymph of $B$. mori larvae. Here, we investigated whether the inhibitor was proteinaceous, non-proteinaceous, or 
both, and documented the changes in the inhibitory activities of membrane-bound and soluble trehalases through the fifth instar and early pupal period.

\section{MATERIALS AND METHODS}

Animals. Larvae of the silkworm B. mori (Kinshu $\times$ Showa) were reared on an artificial diet (Silkmate 2M, Nihon Nosan Kogyo, Yokohama, Japan) at $25^{\circ} \mathrm{C}$ under a $12 \mathrm{~h}$ light: $12 \mathrm{~h}$ dark regime (Sakurai, 1983, 1984). The larval stages were determined as previously described (Sakurai et al., 1998).

Trehalase activity assay. The midguts of Day 0 fifth-instar larvae were excised, cut longitudinally to flush out the gut contents, and washed well with cold Ringer's solution. After blotting with filter paper, the midguts were homogenized in cold $20 \mathrm{~mm}$ phosphate buffer (PB), $\mathrm{pH}$ 6.0, with glassglass homogenizer, followed by sonication for $30 \mathrm{~s}$. The homogenates were then centrifuged at $105,000 \times \mathrm{g}$ at $4^{\circ} \mathrm{C}$ for $60 \mathrm{~min}$. The supernatants and precipitates were used as the fractions containing soluble trehalase and membrane-bound trehalase, respectively. The supernatants were subjected directly to a trehalase activity assay, and the precipitates were suspended in PB for the assay. The amount of protein in each enzyme sample was determined before the trehalase assay using the protein-dye binding method (Bio-rad, Hercules, CA) with bovine serum albumin as a standard protein. Trehalase activity was based on the rate of glucose generation from trehalose. The reaction mixture $(250 \mu \mathrm{l})$ consisted of $62.5 \mu \mathrm{l} 40 \mathrm{mM}$ trehalose (Sigma, St. Louis, MO) in $20 \mathrm{~mm} \mathrm{~PB}, 10 \mu 1$ soluble or membrane-bound trehalase fraction, and $177.5 \mu \mathrm{l} \mathrm{PB}$. The mixture was incubated at $37^{\circ} \mathrm{C}$ for $60 \mathrm{~min}$, and the reaction was stopped by heating in boiling water for $5 \mathrm{~min}$. Coagulated protein was removed by centrifugation at $12,000 \times g$ for $10 \mathrm{~min}$ at $4^{\circ} \mathrm{C}$, and an aliquot of the supernatant was used for measuring the glucose amount using the hexokinase-glucose-6-phosphate dehydrogenase method according to the modified procedure of Bergmeyer et al. (1974) and Knuesel et al. (1998). The reaction was performed in a 1-ml reaction mixture containing 50 units hexokinase, 100 units glucose-6phosphate dehydrogenase, $2 \mathrm{mM}$ NADP, and 2.8 mM ATP (Roche Diagnostics GmbH, Mannheim, Germany). Trehalase activity was determined with reference to a calibration curve depicted using glu- cose (Sigma), and the results were expressed in $\mu \mathrm{mol}$ glucose $/ \mathrm{min} / \mathrm{mg}$ protein.

Trehalase inhibitor extraction. Hemolymph was added to a chilled $1.5-\mathrm{ml}$ plastic tube containing a small amount of phenylthiourea powder, and centrifuged at $10,000 \times g$ for $5 \mathrm{~min}$ at $4^{\circ} \mathrm{C}$ to remove hemocytes. For trehalase inhibitor$\mathrm{M}$, a methanol-extractable inhibitor, hemolymph $(200 \mu \mathrm{l})$ was diluted with distilled water $(400 \mu \mathrm{l})$, boiled for $10 \mathrm{~min}$, chilled on ice, and centrifuged at $15,000 \times g$ for $10 \mathrm{~min}$. The supernatant was mixed with 4 volumes ice-cold methanol, kept at $4^{\circ} \mathrm{C}$ for $2 \mathrm{~h}$, and centrifuged at $15,000 \times \mathrm{g}$ for $10 \mathrm{~min}$ to remove precipitates. The supernatant was transferred to a new tube, to which distilled water was added to reach $80 \%$ methanol concentration, and then the same volume of hexane was added. The tube was mixed vigorously and centrifuged briefly to separate the aqueous methanol and hexane layers, and then the lower layer was transferred to a new tube. The solvent was evaporated using a centrifugal evaporator. The residue was dissolved in $\mathrm{PB}$ of the same volume as the volume of hemolymph, and was used for the inhibitor assay. For trehalase inhibitor-P, a proteinaceous inhibitor, hemolymph was dialyzed with 500 volumes distilled water at $4^{\circ} \mathrm{C}$ for $24 \mathrm{~h}$ using a dialysis tube with $14 \mathrm{kDa}$ cut off (Nacalai Tesque, Kyoto, Japan) and two water changes. The turbid dialysate was centrifuged at $10,000 \times g$ to yield a clear supernatant, and was used for the inhibitor assay. The dialysate was concentrated for further experiments using an ultrafiltration apparatus with a $10 \mathrm{kDa}$ cut off (Centricon YM10, Millipore, Bedford, MA, USA).

Trehalase inhibitor assay. Trehalase inhibitor solution $(137.5 \mu \mathrm{l})$ was mixed with $10 \mu \mathrm{l}$ soluble or membrane-bound trehalase fraction and incubated at $37^{\circ} \mathrm{C}$ for $10 \mathrm{~min}$. Then, $62.5 \mu \mathrm{l}$ of $40 \mathrm{~mm}$ trehalose were added, adjusted to a volume of $250 \mu \mathrm{l}$ with $\mathrm{PB}$, and incubated at $37^{\circ} \mathrm{C}$ for $60 \mathrm{~min}$. When hemolymph was used directly for assessing the inhibitory activity, $137.5 \mu \mathrm{l}$ of hemolymph were added to the reaction mixture instead of trehalase inhibitor. The reaction was stopped by heating in boiling water for $5 \mathrm{~min}$. The mixture was centrifuged at $12,000 \times g$ for $10 \mathrm{~min}$ at $4^{\circ} \mathrm{C}$, and an aliquot of the supernatant was used for assessing glucose content as described above. The amounts of glucose in the inhibitor extracts or hemolymph were determined in the same manner. The amount 
of the released glucose in each assay was then calculated by subtracting the amount of glucose in the inhibitor sample from that in the mixture after the reaction.

Treatment with trypsin. Inhibitor preparations $(150 \mu \mathrm{l})$ were added to $10 \mu \mathrm{l}$ trypsin in $\mathrm{PB}$ $(10 \mathrm{mg} / \mathrm{ml}$, Nacalai Tesque), and the solution was incubated for $5 \mathrm{~h}$ at $37^{\circ} \mathrm{C}$. The reaction was stopped by adding $10 \mu \mathrm{l}$ trypsin inhibitor $(10 \mathrm{mg} / \mathrm{ml}$, soybean trypsin inhibitor, Sigma). The trypsin-treated solution was centrifuged at $10,000 \times g$ at $4^{\circ} \mathrm{C}$ for $5 \mathrm{~min}$, and the supernatant was used immediately for the trehalase inhibitor assay.

\section{RESULTS}

Enzymatic activities of soluble and membranebound trehalases of Day 0 larvae

The midguts of Day 0 fifth-instar larvae were extracted and used as the enzyme source. We first determined the optimal incubation time for trehalase activity assay. For both types of trehalase, glucose levels increased significantly from 1 to $4 \mathrm{~h}$ of incubation (Fig. 2). Based on these results, we used $1 \mathrm{~h}$

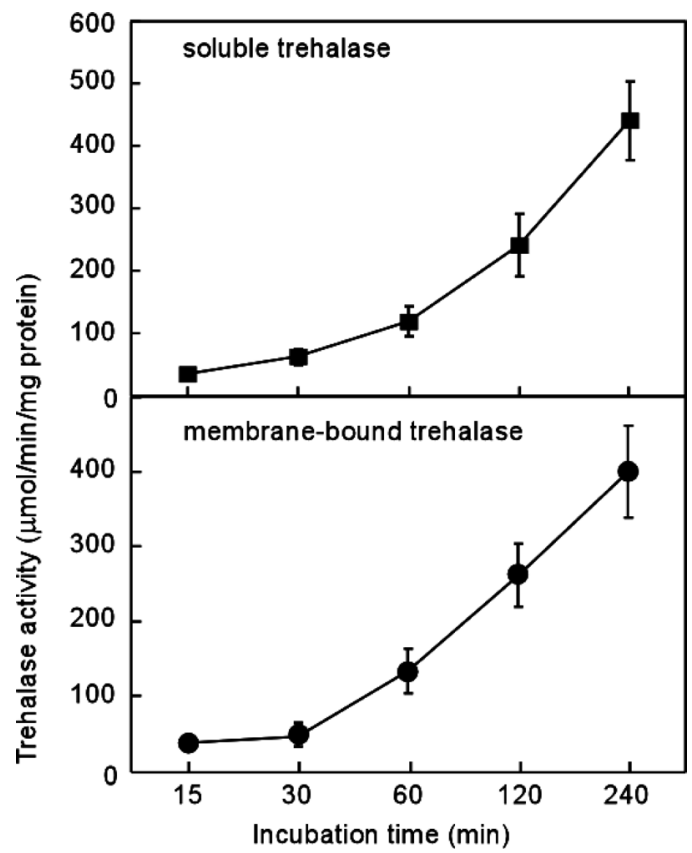

Fig. 2. Time-course of glucose generation by soluble and membrane-bound trehalase in the midguts of $B$. mori larvae. Trehalase was prepared from Day 0 fifth-instar larvae and incubated individually with trehalose for the indicated period. The enzyme activities are expressed as $\mu$ mol glucose produced by $1 \mathrm{mg}$ protein. Each value is mean $\pm \mathrm{SD}(n=3)$. as the incubation period for all experiments. Two types of trehalase inhibitor in the hemo-
lymph

The hemolymph of Day 3 fifth-instar larvae was incubated with soluble and membrane-bound trehalases (Fig. 3). In the standard assay condition, the membrane-bound trehalase activity was 148.7 $\mu \mathrm{mol} / \mathrm{min} / \mathrm{mg}$ protein, which was higher than the soluble trehalase activity of $52.1 \mu \mathrm{mol} / \mathrm{min} / \mathrm{mg}$ protein. For both types, the amount of released glucose was much smaller when incubated with larval hemolymph than in assays without hemolymph (0.9 and $7.4 \mu \mathrm{mol} / \mathrm{min} / \mathrm{mg}$ protein, respectively). That is, adding hemolymph inhibited the activity of soluble trehalase by $95 \%$ and membrane-bound trehalase by $98.3 \%$.

To characterize these inhibitors, we tested differ-

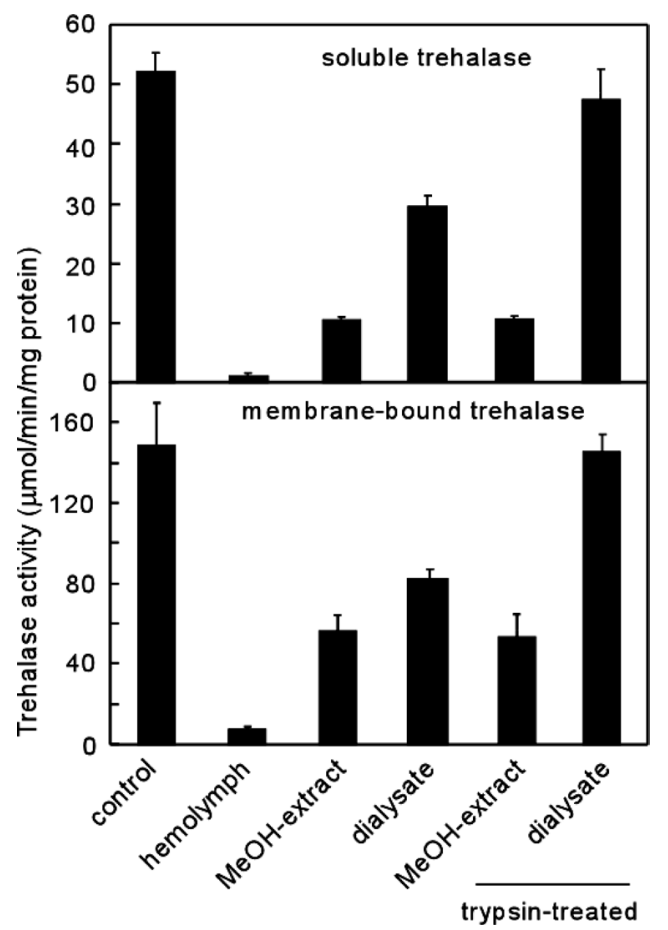

Fig. 3. Larval hemolymph contains two types of trehalase inhibitor. The inhibitory activity was measured separately on soluble (upper panel) and membrane-bound trehalases (lower panel) prepared from Day 0 fifth-instar larvae. Each type of trehalase was incubated individually with the following samples for $1 \mathrm{~h}$ : hemolymph, hemolymph from Day 3 fifth-instar larvae; MeOH-extract, methanol extracts of the same hemolymph; dialyzed, dialyzed hemolymph in water; trypsintreatment, methanol-extracted hemolymph and dialyzed hemolymph treated with trypsin and then subjected to an enzyme assay. Trehalase activity is expressed as $\mu \mathrm{mol}$ glucose $/ \mathrm{min} / \mathrm{mg}$ protein. Each value is mean $\pm \mathrm{SD}(n=3)$. 
ent extraction methods and dialysis in distilled water. Methanol extracts elicited inhibitory activity on both soluble and membrane-bound trehalase (by $80 \%$ and $62.5 \%$, respectively). The inhibitor was extractable with ethanol as well but not with $n$-butanol (data not shown). The inhibitor was recovered from hemolymph that was heated in boiling water prior to methanol extraction (see Materials and Methods). In addition, methanol extractions of the dialysate from the dialysis did not exhibit inhibitor activity (data not shown), indicating that the methanol-extractable inhibitor was not proteinaceous.

However, the dialysate itself did show inhibitory activity. Because we used a dialysis tube with a $14 \mathrm{kDa}$ cut off, the inhibitor molecules in the dialysate may have been large, and were probably proteinaceous molecules. To examine this possibility, we treated methanol extracts of hemolymph and the dialysate with trypsin (Fig. 3). Trypsin treatment did not reduce the inhibitory activity of methanol extracts of either soluble (10.1 and $10.4 \mu \mathrm{mol} / \mathrm{min} / \mathrm{mg}$ protein before and after treatment, respectively) or membrane-bound trehalase (55.7 and $46.8 \mu \mathrm{mol} / \mathrm{min} / \mathrm{mg}$ protein before and after treatment, respectively). In contrast, trypsin treatment of the dialyzed hemolymph abolished most inhibitory activity. The soluble and membrane-bound trehalase activities were $91 \%$ and
$98 \%$ of the average activities determined without the inhibitor fraction, respectively, but the difference was not significant, showing that the inhibitor may have been protein. The slightly reduced activities may have been caused by a partial degradation of the trehalases by trypsin even in the presence of trypsin inhibitor. Thus, the hemolymph of B. mori larvae contained two types of trehalase inhibitors, a methanol-extractable inhibitor (hereafter, inhibitor$\mathrm{M})$ and a proteinaceous inhibitor (inhibitor-P).

\section{Effects of the two trehalase inhibitors on treha- lase activity}

We analyzed the concentration-dependent inhibitory activity of trehalase inhibitor-M and -P on soluble trehalase activity in the midgut. Both samples were prepared from Day 3 fifth-instar larvae, and their concentrations were adjusted to one hemolymph equivalent, and then serially diluted or concentrated and added to the reaction mixture for the assay (Fig. 4). Trehalase activity decreased in both samples in a concentration-dependent manner with a dilution magnitude for $50 \%$ inhibition $\left(\mathrm{IC}_{50}\right)$ of 0.094 for inhibitor-M and 1 for inhibitor-P, showing that the inhibitory activity of inhibitor-M was much higher than that of inhibitor-P. In addition, inhibitor-M in non-diluted hemolymph $(\times 1$ sample) inhibited about $80 \%$ of trehalase activity, and inhibitor-P elicited about 50\% inhibition, indi-

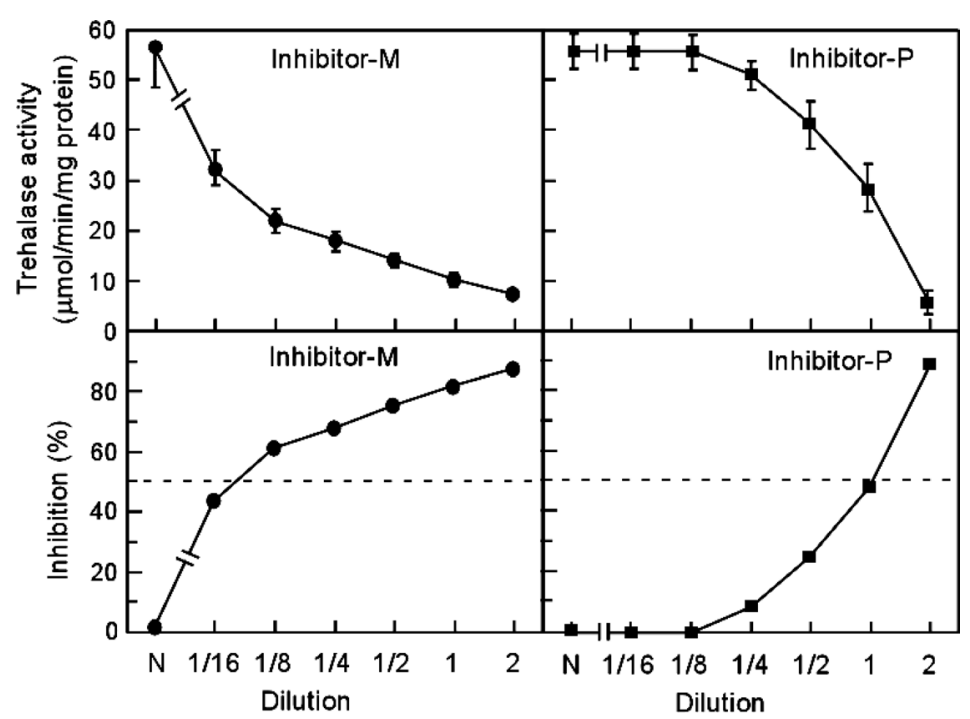

Fig. 4. Concentration-dependent inhibitory effects of trehalase inhibitors on soluble trehalase activity in the midgut. Inhibitor$\mathrm{M}$ and -P were prepared from the hemolymph of Day 3 fifth-instar larvae and brought to a concentration of twice that of the hemolymph, and inhibitory activities in serially diluted inhibitor samples were measured on soluble trehalase. Upper panel, trehalase activity expressed as in Fig. 3. Lower panel, percent inhibition of trehalase activity where a value of $100 \%$ indicates no glucose generation. Each value is mean $\pm \mathrm{SD}(n=3)$. 
cating that soluble trehalase activity may be completely inhibited by a combination of the two types of inhibitor.

\section{Developmental changes in the inhibitory activi- ties of the two types of trehalase inhibitor}

We measured the changes in the inhibitory activities of trehalase inhibitor-M and -P in the hemolymph throughout the fifth larval instar and early pupal period (Fig. 5). Hemolymph was collected every day, and inhibitor activities were measured separately for the two types of trehalase. Inhibitor-M elicited $60-70 \%$ inhibition of membrane-bound trehalase between Day 0 and Day 4, and rapidly decreased to a very low level on Day 5 and thereafter. Inhibitor-M inhibited soluble trehalase at a fairly constant level until Day 4, declined to approximately $20 \%$ inhibition on Day 5 , remained at this level until Day 8, and then gradually declined to an almost undetectable level on the day of pupation.

Inhibitor-P exhibited a low level of inhibition toward soluble trehalase on Day 0 to Day 2 of the fifth instar, and then increased gradually from Day 3 to the maximal level ( $80 \%$ inhibition) on Day 7, the day of gut purge. The activity dropped greatly to no inhibition on Day 9, remained undetectable through Day 10, then peaked at pupation and decreased gradually until Day 3 of the pupal stage. Inhibitor-P exhibited a similar pattern for membrane-bound trehalase, except during the early fifth instar and on Day 8, 1 day after gut purge. The inhibitory activity was low on Day 0 , as for soluble trehalase, but increased markedly (80-90\% inhibition) from Day 1 of the fifth instar, remained high until Day 8, and decreased to an undetectable level on Days 9 and 10; it then increased transiently on the day of pupation to $87 \%$ inhibition and decreased thereafter as observed for soluble trehalase.

The amount of each inhibitor prepared on a given day was the same between the assays of soluble and membrane-bound trehalase, and we used the same enzyme preparation throughout the study. Accordingly, we could estimate the sensitivity of each trehalase to both types of inhibitor. For example, inhibition by inhibitor-P prepared from Day 2 hemolymph shows that membrane-bound trehalase is more sensitive to inhibitor-P than soluble trehalase is. Similarly, inhibitor-M prepared from Day 6 hemolymph elicited a slightly stronger inhibition

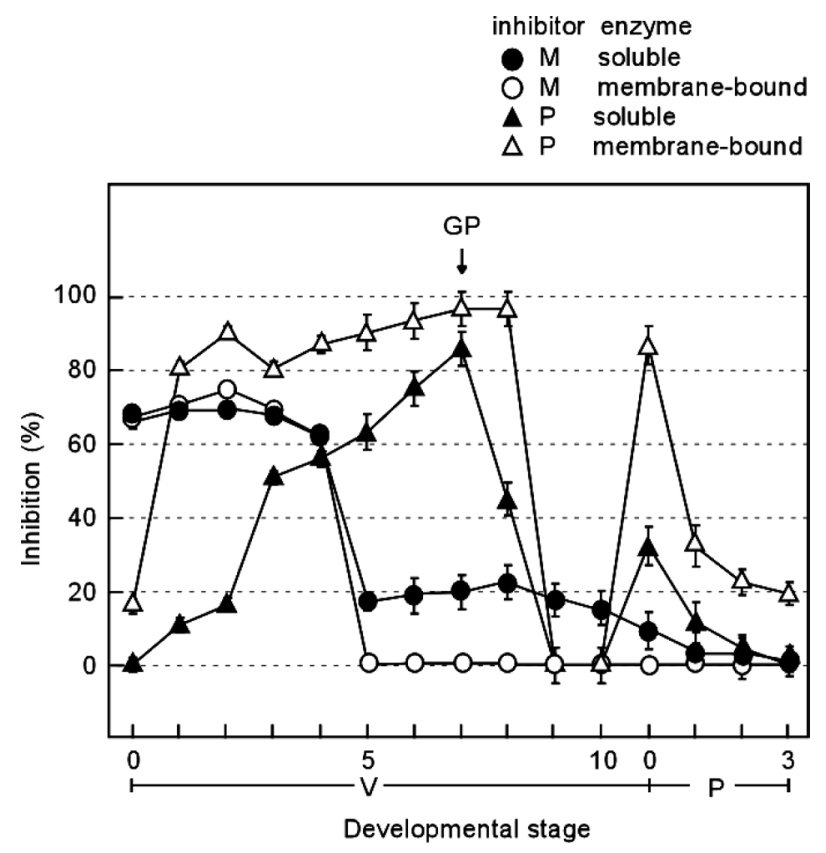

Fig. 5. Developmental changes in the inhibitory activities of trehalase inhibitor-M and -P through the fifth instar and early pupal period. We prepared the two types of inhibitors from hemolymph every day, adjusted their concentrations to the same as that of the hemolymph, and assessed their inhibitory activities on soluble and membrane-bound trehalases prepared from the midguts of Day 0 fifth-instar larvae. Closed and open circles, inhibitory activities of trehalase inhibitor-M on soluble and membrane-bound trehalase, respectively; closed and open triangles, inhibitory activities of trehalase inhibitor-P on soluble and membrane-bound trehalase, respectively; GP, gut purge. Inhibitory activities are expressed as in Fig. 4. Each value is mean $\pm \mathrm{SD}(n=3)$. Where not shown, the SD bars are smaller than the symbol size.

toward membrane-bound trehalase than soluble trehalase.

Inhibitory activity in extracts of an artificial diet

Mulberry leaves contain sugar-mimic alkaloids that inhibit trehalase (Hirayama et al., 2007). Because the artificial diet we used for rearing $B$. mori larvae contained mulberry leaf powder (percentage not indicated by the producer), we extracted the diet ( $1 \mathrm{~g}$ wet weight containing $23.8 \%$ of dry diet powder) with methanol and examined its inhibitory activity. The extract exhibited $85 \%$ inhibition of soluble trehalase activity in the midgut. 


\section{DISCUSSION}

\section{Two types of trehalase inhibitor and two types of trehalase}

We found two types of trehalase inhibitor in the larval and pupal hemolymph of B. mori. Inhibitor$M$ was extractable with methanol and ethanol. Treatment with butanol and hexane did not abolish the activity, showing that trehalase-M is resistant to organic solvents. It was also heat stable (see Materials and Methods), did not remain in the dialysate after dialysis, and exhibited strong resistance against digestion by trypsin. These physical properties are different from other inhibitors thus far identified in insects: trehalase inhibitor is heat-labile and nondialyzable in only two other insect species, the blowfly $P$. regina (Friedman, 1961) and the cockroach $P$. americana (Hayakawa et al., 1989).

Plants contain various sugar-metabolizing enzyme inhibitors such as $\beta$-glucosides (Silva et al., 2006) and sugar-mimic alkaloids (Hirayama et al., 2007) as a defense against herbivorous insects. Leaves of mulberry, the specific host plant of $B$. mori, contain the sugar-mimic alkaloids with potent inhibitory activity of soluble trehalase and membrane-bound trehalase (Hirayama et al., 2007). Although it is not known in B. mori whether dietary trehalase inhibitors are absorbed from the gut lumen into the hemolymph, it is possible, as the alkaloids are absorbed in S. cynthia larvae (Hirayama et al., 2007). In addition, the intensity of inhibitory activity appears to be correlated with the feeding activity of the larvae. The inhibitory activity of inhibitor-M was high from the early to the middle feeding period (Days 0-4), then decreased dramatically to a low level during the late feeding period and after feeding ceased. The feeding activity is high until Day 5 and decreases before the onset of wandering on Day 6. We therefore assume that this inhibitor originated from mulberry leaves and could be the same as the sugar-mimic alkaloids, although it remains to be determined if the dietary trehalase inhibitors are absorbed in B. mori larvae.

Inhibitor-P was heat-labile, remained in the dialysate after dialysis, and was inactivated by trypsin treatment, indicating that it is a protein. It may be similar to the proteinaceous $86-\mathrm{kDa}$ inhibitor that reduces trehalase activity in $P$. ameri- cana (Hayakawa et al., 1989). It inhibited the enzymatic activities of membrane-bound and soluble trehalases equally.

The activities of inhibitor-M and -P may be regulated differently. Inhibitor-M activity decreased sharply on Day 5, and hemolymph ecdysteroid concentration begins to increase on Day 5 (Sakurai et al., 1998), an indication that a small increase in ecdysteroid concentration on Day 5 could affect the concentration of inhibitor-M in hemolymph, although this remains to be investigated. Developmental changes in inhibitor-P may be caused by the changing hemolymph ecdysteroid titer. Inhibitor-P activity was at the lowest level on Day 9, followed by a large increase on the day of pupation and a decrease thereafter. During this period, hemolymph ecdysteroid titer peaks on Day 8 in preparation for pupation, drops during pupation, and then increases again before adult differentiation (Mizoguchi et al., 2001). In the bamboo borer $O$. fuscidentalis, exogenous $20 \mathrm{E}$ decreases the inhibitory activity of a proteinaceous trehalase inhibitor in larval hemolymph (Tatun et al., 2008a). If this is also the case in $B$. mori, the high $20 \mathrm{E}$ concentration before pupation may decrease inhibitor-P concentration, while low 20E concentrations during feeding and at pupation may allow the inhibitor to increase.

\section{Possible roles of trehalase inhibitors and treha- lases in the regulation of hemolymph trehalase concentration}

Changes in hemolymph trehalose concentration have been linked to trehalose production by the fat body, the principle tissue that secretes trehalose (Murphy and Wyatt, 1965; Hirano and Yamashita, 1983; Becker et al., 1996), and trehalose metabolism by trehalase (Yamashita et al., 1974; Friedman, 1978; Becker et al., 1996). These factors could account for the decline in trehalose concentration at the beginning of the prepupal period, as soluble trehalase activity increases (see Fig. 1), coupled with the decline of trehalose production by fat bodies (Hirano and Yamashita, 1983). In contrast, trehalose concentration during the feeding period remains high despite the substantial levels of membrane-bound trehalase activity in tissues (Yamashita et al., 1974), and thus a proper explanation for the regulation of trehalose concentration during feeding has not yet been proposed. 
Our results may suggest that in addition to the activity of two types of trehalase (membranebound and soluble; Yamashita et al., 1974) and trehalose production (Hirano and Yamashita, 1983), two different trehalase inhibitors are involved in the regulation of hemolymph trehalose. Coordination of these five factors may account for the changes in trehalose concentration throughout the fifth instar and early pupal period, which may be divided into four periods (Fig. 1). The first period is from the fifth instar to Day 5, during which larvae actively feed and both trehalose production by fat bodies and trehalose synthase activity increase five-fold (Hirano and Yamashita, 1983). During this period, the net amount of trehalose in the hemolymph increases four- to six-fold (Oda et al., 1997), despite high membrane-bound trehalase activity with peak activity on Days 3 and 4, indicating that metabolism of trehalose is suppressed at a minimum level. Diet-origin inhibitor-M may be the primary inhibitor of membrane-bound trehalase activity (see Fig. 5). As a result, hemolymph trehalose concentration may remain stable at a high level.

The second period is from Day 5 to 7 , the day of gut purge, during which hemolymph trehalose concentration declines from $10 \mathrm{~mm}$ to about $2 \mathrm{mM}$. Hemolymph trehalose concentration decreases despite the presence of inhibitor-P, whose concentration is sufficient to strongly inhibit both types of trehalase. This decrease may be due to soluble trehalase, which increases in activity on Day 5 and peaks on Day 6, together with a sharp decline in trehalose synthase activity before the onset of spinning (Hirano and Yamashita, 1983). Greater levels of ecdysteroids in the hemolymph on these days may up-regulate soluble trehalase gene expression followed by an increase in soluble trehalase activity, as demonstrated in O. fuscidentalis (Tatun et al., 2008b). The increased soluble trehalase activity may overcome inhibitor-P inhibition, causing the sharp decrease in trehalose concentration in conjunction with the cessation of sugar-mimic alkaloid consumption due to the cessation of feeding.

The third period is from Day 7 to the day of pupation, during which trehalose concentration displays a broad peak. From Day 7 to 9, inhibitor-P activity decreases sharply, and a simultaneous increase in soluble trehalase activity occurs. Although trehalose production by the fat bodies de- clines during this period, produced trehalose may accumulate in the hemolymph due to low trehalose metabolism: the lower activity of soluble trehalase from Day 7 to 8 and the presence of inhibitor-M in the hemolymph may contribute to protect the hydrolysis of trehalose.

The fourth period is the pharate pupal (Day 10) and early pupal period. Trehalose concentration decreases and then increases after pupation. Soluble trehalase activity greatly increases, while inhibitor$\mathrm{P}$ peaks at pupation. The decrease in trehalose is rather small despite the large increase in soluble trehalase activity, which may be explained by the large increase in inhibitor-P activity. However, we are unable to interpret the increase in trehalose after pupation under increasing soluble trehalase activity and decreasing inhibitor-P activity. During this period, both trehalose production by fat bodies and trehalose synthase activity in fat bodies are very low (Hirano and Yamashita, 1983), indicating that trehalose production by fat bodies may not contribute to the increase in hemolymph trehalose, and therefore the regulation of trehalose concentration remains to be elucidated.

The regulation of dynamic changes in hemolymph trehalose concentration through larval and pupal periods may involve various factors: inhibitor-M and inhibitor-P; two types of trehalases and their sensitivities to the two inhibitors; expression of two trehalase genes, the soluble trehalase gene treh-1 and the membrane-bound trehalase gene treh-2 (Mitsumasu et al., 2006; Tatun et al., 2008b); and trehalose production by trehalose synthase. Among these factors, the identification of the inhibitor-P gene and regulation of its expression are necessary to better understand the regulatory mechanisms of hemolymph trehalose concentration.

\section{ACKNOWLEDGEMENTS}

This study was supported by the Staff Development Project from the Thai Commission on Higher Education, Thailand, to N.T.

\section{REFERENCES}

Azuma, M. and O. Yamashita (1985) Cellular localization and proposed function of midgut trehalase in the silkworm larva, Bombyx mori. Tissue Cell 17: 539-551.

Becker, A., P. Schlöder, J. E. Steele and G. Wegener (1996) The regulation of trehalose metabolism in insects. Experientia 52: 433-439. 
Bergmeyer, H. U., E. Bernt, F. Schmidt and H. Stork (1974) D-Glucose determination and glucose-6-phosphate dehydrogenase. In Methods of Enzymatic Analysis. Vol. 3 (H. U. Bergmeyer, ed.). Academic Press, New York, pp. 1196-1201.

Blatt, J. and F. Roces (2001) Haemolymph sugar levels in foraging honeybees (Apis mellifera Carnica): Dependence on metabolic rate and in vivo measurement of maximal rates of trehalose synthesis. J. Exp. Biol. 204: 2709-2716.

Chino, H., R. G. H. Downer, G. R. Wyatt and L. I. Gilbert (1981) Lipophorin, a major class of lipoprotein of insect hemolymph. Insect Biochem. 11: 491.

Friedman, S. (1961) Inhibition of trehalase activity in the hemolymph of Phormia regina Meig. Arch. Biochem. Biophys. 93: 550-554.

Friedman, S. (1978) Trehalose regulation, one aspect of metabolic homeostasis. Ann. Rev. Entomol. 23: 389407.

Gäde, G., K. H. Hoffmann and J. H. Spring (1997) Hormonal regulation in insects: facts, gaps, and future directions. Physiol. Rev. 77: 963-1032.

Goto, M., K. Takahashi and C. Suzuki (1993) Ecological study on the barnyard grass stem borer, Enosima leucotaeniella (Ragonot) (Lepidoptera: Pyralidae) VIII. Seasonal changes of carbohydrate contents in overwintering larvae. Appl. Entomol. Zool. 28: 417-421.

Hayakawa, Y., A. P. Jahagirdar, M. Yaguchi and R. G. H. Downer (1989) Purification and characterization of trehalase inhibitor from hemolymph of the American cockroach, Periplaneta americana. J. Biol. Chem. 264: 16165-16169.

Hirano, M. and O. Yamashita (1983) Developmental changes in trehalose biosynthesis in fat body of the silkworm, Bombyx mori: trehalose synthase related to regulation of hemolymph trehalose during metamorphosis. Insect. Biochem. 13: 593-599.

Hirayama, C., K. Konno, N. Wasano and M. Nakamura (2007) Differential effects of sugar-mimic alkaloids in mulberry latex on sugar metabolism and disaccharidases of Eri and domesticated silkworms: enzymatic adaptation of Bombyx mori to mulberry defense. Insect Biochem. Molec. Biol. 37: 1348-1358.

Kikawada, T., A. Saito, Y. Kanamori, Y. Nakahara, K. Iwata, D. Tanaka, M. Watanabe and T. Okuda (2007) Trehalose transporter 1, a facilitated and high-capacity trehalose transporter, allows exogenous trehalose uptake into cells. Proc. Natl. Acad. Sci. USA 104: 1158511590 .

Knuesel, I., S. Murao, T. Shin, T. Amachi and H. Kayser (1998) Comparative studies of suidatrestin, a specific inhibitor of trehalases. Comp. Biochem. Physiol. B 120: 639-646.

Miller, S. G., R. F. Leclerc, S. J. Seo and C. Malone (1990) Synthesis and transport of storage proteins by testes in Heliothis virescens. Arch. Insect Biochem. Physiol. 14: 151-170.

Mitsumasu, K., M. Azuma, T. Niimi, O. Yamashita and T. Yaginuma (2005) Membrane penetrating trehalase from silkworm Bombyx mori molecular cloning and localization in larval midgut. Insect Mol. Biol. 14: 501-508.

Mitsumasu, K., M. Azuma, T. Niimi, O. Yamashita and T. Yaginuma (2006) Diapause hormone-enhanced expression of the trehalase-2 gene encoding a novel membrane-penetrating type in Bombyx mori developing ovaries and the gene structure. J. Insect Biotechnol. Sericol. 75: 71-77.

Mizoguchi, A., Y. Ohashi, K. Hosoda, J. Ishibashi and H. Kataoka (2001) Development profile of the changes in the prothoracicotropic hormone titer in hemolymph of the silkworm Bombyx mori: correlation with ecdysteroid secretion. Insect Biochem. Molec. Biol. 31: 349-358.

Mullins, D. E. (1985) Chemistry and physiology of the hemolymph. In Comprehensive Insect Physiology, Biochemistry and Pharmacology. Vol. 3 (G. A. Kerkut and L. I. Gilbert, eds.). Pergamon Press, Oxford, pp. 355-400.

Murphy, T. A. and G. R. Wyatt (1965) The enzymes of glycogen and trehalose synthesis in silk moth fat body. J. Biol. Chem. 240: 1500-1508.

Oda, Y., M. Iwami, M. Osani and S. Sakurai (1997) Dynamics of haemolymph sorbitol-6-phosphate and its control by ecdysteroid in the larvae of the silkworm, Bombyx mori. Insect Biochem. Molec. Biol. 27: 461-468.

Oda, Y., M. Uejima, M. Iwami and S. Sakurai (2000) Role of ecdysteroids in the dynamics of insect haemolymph sugar. Zool. Sci. 17: 785-789.

Ogiso, M. and S. Y. Takahashi (1984) Trehalases from the male accessory glands of the American cockroach: developmental changes and the hormonal regulation of the enzymes. Gen. Comp. Endocrinol. 55: 387-392.

Sakurai, S. (1983) Temporal organization of endocrine events underlying larval-larval ecdysis in the silkworm, Bombyx mori. J. Insect Physiol. 29: 919-932.

Sakurai, S. (1984) Temporal organization of endocrine events underlying larval-pupal metamorphosis in the silkworm, Bombyx mori. J. Insect Physiol. 30: 657-664.

Sakurai, S., S. Satake and M. Kaya (1998) Hemolymph ecdysteroid titer and ecdysteroid-dependent developmental events in the last-larval stadium of the silkworm, Bombyx mori: role of low ecdysteroid titer in larval-pupal metamorphosis and a reappraisal of the head critical period. J. Insect Physiol. 44: 867-881.

Silva, M. C. P., R. W. Terra and C. Ferreira (2006) Absorption of toxic $\beta$-glucosides produced by plants and their effect on tissue trehalases from insects. Comp. Biochem. Physiol. B 143: 367-373.

Singtripop, T., Y. Oda, S. Wanichacheewa and S. Sakurai (2002) Sensitivities to juvenile hormone and ecdysteroid in the diapause larvae of Omphisa fuscidentalis based on the hemolymph trehalose dynamic index. $J$. Insect Physiol. 48: 817-824.

Steele, J. E. and T. Paul (1985) Corpus cardiacum stimulated trehalose efflux from cockroach (Periplaneta americana) fat body: control by calcium. Can. J. Zool. 63: 63-66.

Su, Z. H., Y. Sato and O. Yamashita (1993) Purification, cDNA cloning and Northern blot analysis of trehalase of pupal midgut of the silkworm, Bombyx mori. Biochim. Biophys. Acta 1173: 217-224.

Sumida, M. and O. Yamashita (1983) Purification and some 
properties of soluble trehalase from midgut of pharate adult of the silkworm, Bombyx mori. Insect Biochem. 13: 257-265.

Tatun, N., T. Singtripop and S. Sakurai (2008a) Dual control of midgut trehalase activity by 20-hydroxyecdysone and an inhibitory factor in the bamboo borer Omphisa fuscidentalis Hampson. J. Insect Physiol. 54: 351-357.

Tatun, N., T. Singtripop, J. Tungjitwitayakul and S. Sakurai (2008b) Regulation of soluble and membrane-bound trehalase activity and gene expression of the enzyme in the larval midgut of the bamboo borer Omphisa fuscidentalis. Insect Biochem. Molec. Biol. doi: 10.1016/j. ibmb.2008.05.003

Thompson, S. N. (2003) Trehalose-the insect 'blood' sugar. Adv. Insect Physiol. 31: 203-285.
Wegener, G., V. Tschiedel, P. Schlöder and O. Ando (2003) The toxic and lethal effects of trehalase inhibitor trehazolin in locusts are caused by hypoglycemia. J. Exp. Biol. 206: 1233-1240.

Wyatt, G. R. (1967) The biochemistry of sugars and polysaccharides in insects. Adv. Insect Physiol. 4: 287-360.

Yaginuma, T. and G. M. Happ (1989) 20-Hydroxyecdysone acts in the male pupa to commit accessory glands toward trehalase production in the adult mealworm beetle (Tenebrio molitor). Gen. Comp. Endocrinol. 73: 173-185.

Yamashita, O., M. Sumida and K. Hasegawa (1974) Developmental changes in midgut trehalase activity and its localization in the silkworm, Bombyx mori. J. Insect Physiol. 20: 1079-1085. 